\title{
Geometry of periodic monopoles
}

\author{
R. Maldonado* and R. S. Ward ${ }^{\dagger}$ \\ Department of Mathematical Sciences, Durham University, Durham DH1 3LE, United Kingdom
}

(Received 6 October 2013; published 6 December 2013)

\begin{abstract}
Bogomol'nyi-Prasad-Sommerfield monopoles on $\mathbb{R}^{2} \times S^{1}$ correspond, via the generalized Nahm transform, to certain solutions of the Hitchin equations on the cylinder $\mathbb{R} \times S^{1}$. The moduli space $\mathcal{M}$ of two monopoles with their center of mass fixed is a four-dimensional manifold with a natural hyperkähler metric, and its geodesics correspond to slow-motion monopole scattering. The purpose of this paper is to study the geometry of $\mathcal{M}$ in terms of the Nahm-Hitchin data, i.e., in terms of structures on $\mathbb{R} \times S^{1}$. In particular, we identify the moduli, derive the asymptotic metric on $\mathcal{M}$, and discuss several geodesic surfaces and geodesics on $\mathcal{M}$. The latter include novel examples of monopole dynamics.
\end{abstract}

DOI: $10.1103 /$ PhysRevD.88.125013

PACS numbers: $11.15 .-\mathrm{q}$

\section{INTRODUCTION}

This paper deals with periodic Bogomol'nyi-PrasadSommerfield (BPS) monopoles, namely, Yang-MillsHiggs fields $\left(\Phi, A_{j}\right)$ on $\mathbb{R}^{2} \times S^{1}$ satisfying the Bogomolny equations. A useful tool for understanding systems of this type is the generalized Nahm transform. The best-known Nahm transform describes BPS monopoles on $\mathbb{R}^{3}$ in terms of solutions of a set of ordinary differential equations, namely, the Nahm equations. But this is part of a more general picture (which also includes the Atiyah-DrinfeldHitchin-Manin (ADHM) construction of self-dual YangMills instantons): a generalized Nahm transform, which may be understood in terms of the reciprocity between selfdual Yang-Mills equations on dual 4-tori [1,2]. Suitable rescalings of the tori (and corresponding dimensional reductions of the self-duality equations) then give, as special cases, the ADHM transform and the Nahm transform for monopoles on $\mathbb{R}^{3}$, as well as several other systems including the present one. The general scheme suggests that the Nahm transforms of BPS monopoles on $\mathbb{R}^{2} \times S^{1}$ are solutions of the Hitchin equations on the cylinder $\mathbb{R} \times S^{1}$ (satisfying appropriate boundary conditions), where the two circles are dual to each other. This was confirmed in [3], where many of the details were worked out.

In this paper, we focus on the case of periodic twomonopole fields with gauge group $\mathrm{SU}(2)$. Such fields may also be visualized as a pair of infinite monopole chains. By contrast with the case of monopoles in $\mathbb{R}^{3}$, the system as a whole is infinitely massive, so its center of mass and overall phase are parameters which must be kept fixed [4]. However, the relative separation and phase of the monopoles are free to vary. So we focus on the relative

\footnotetext{
*rafael.maldonado@durham.ac.uk

†richard.ward@durham.ac.uk
}

Published by the American Physical Society under the terms of the Creative Commons Attribution 3.0 License. Further distribution of this work must maintain attribution to the author(s) and the published article's title, journal citation, and DOI. moduli space $\mathcal{M}$ of solutions: this is a four-dimensional manifold equipped with a natural hyperkähler metric. The geodesics in $\mathcal{M}$ are of particular interest, as they correspond to slow-motion dynamics of the system. Our purpose is to study the geometry of $\mathcal{M}$ in the Nahm-transformed picture, i.e., in terms of structures on the cylinder $\mathbb{R} \times S^{1}$. The dynamics of monopoles in this system is different from that of the well-known case of monopoles on $\mathbb{R}^{3}$, owing to the periodicity. Although the metric on $\mathcal{M}$ is not known explicitly, one can identify some geodesics as fixed-point sets of discrete symmetries, and this provides examples of such novel dynamics.

For the space $\mathbb{R}^{2} \times S^{1}$ on which the monopole fields $\left(\Phi, A_{j}\right)$ live, we use coordinates $(x, y, z)$, with $z$ having period $2 \pi$. So the gauge potential $A_{j}$ and the Higgs field $\Phi$ are smooth functions of $(x, y, z)$, periodic in $z$, and they satisfy the Bogomolny equations $2 D_{j} \Phi=-\varepsilon_{j k l} F_{k l}$, where $F_{k l}$ denotes the gauge field. The monopoles are located, roughly speaking, at the zeros of $\Phi$. For monopole fields of charge 2, the boundary behavior in the nonperiodic directions, i.e., as $\rho \rightarrow \infty$ where $x+\mathrm{i} y=\rho \mathrm{e}^{\mathrm{i} \chi}$, is

$$
\begin{aligned}
& \Phi-(\mathrm{i} / \pi)(\log \rho / C) \sigma_{3} \rightarrow 0, \quad A_{x} \rightarrow 0, \\
& A_{y} \rightarrow 0, \quad A_{z}-(\mathrm{i} \chi / \pi) \sigma_{3} \rightarrow 0,
\end{aligned}
$$

locally in some gauge. Here $C$ is a positive constant which determines the monopole size or, more accurately, the ratio between the monopole size and the $z$ period. The $C \rightarrow 0$ limit corresponds to monopoles on $\mathbb{R}^{3}$; the opposite extreme $C \gg 1$, where the monopoles spread out, is discussed in more detail in [5]. Note that the system is not rotationally symmetric about the $z$ axis: this is reflected in the boundary condition for $A_{z}$. It does, however, admit the discrete symmetry of rotations by $\pi$ about the $z$ axis, which can be compensated by a periodic gauge transformation.

Such monopole fields (or, rather, the subset of centered monopole fields) correspond, via the Nahm transform [3], to the following Nahm-Hitchin data on $\mathbb{R} \times S^{1}$. As coordinates on $\mathbb{R} \times S^{1}$, we take $r \in \mathbb{R}$ and $t$ with period 1 . Let $s$ denote the complex coordinate $s=r+\mathrm{i} t$. The fields on 
$\mathbb{R} \times S^{1}$ consist of a gauge potential $\left(a_{r}, a_{t}\right)$ with the gauge group in this case being $\mathrm{SU}(2)$, and a complex Higgs field $\phi$ in the adjoint representation. The gauge field $f_{r t}$ is simply written as $f$. These fields satisfy the Hitchin equations

$$
D_{\bar{s}} \phi=0, \quad 2 f=\mathrm{i}\left[\phi, \phi^{*}\right] .
$$

Here $D_{\bar{s}} \phi:=\partial_{\bar{s}} \phi+\left[a_{\bar{s}}, \phi\right]$, and $\phi^{*}$ denotes the complex conjugate transpose of $\phi$. The field $\phi$ is constrained by

$$
\operatorname{det} \phi=C^{2}(-2 \cosh (2 \pi s)+K),
$$

where $K$ is some complex constant, and the boundary condition is $f \rightarrow 0$ as $r \rightarrow \pm \infty$.

In general, for a periodic $n$-monopole system, the NahmHitchin data would be $\mathfrak{t}(n)$ valued. In our case, $n=2$. The significance of the fields $\left(\phi, a_{\bar{s}}\right)$ being $\mathfrak{s} \mathfrak{u}(2)$ valued, rather than $\mathfrak{u}(2)$ valued, is that the corresponding two-monopole system is centered, with its center of mass fixed at the point $(0,0,0)$. As noted above, we are only interested in the relative separation of the monopoles, and so we restrict to $\mathfrak{S H}(2)$.

Actually, one may equally regard the center of mass as being located at the point $(0,0, \pi)$, since the distinction between these two possibilities is ambiguous in view of the $z$ periodicity. There is a natural map $\tau$ which translates a monopole solution by $\pi$ in the $z$ direction, and which therefore interchanges these two centering points. The corresponding map on $\left(\phi, a_{r}, a_{t}\right)$ consists of gauging by an antiperiodic gauge transformation, i.e., by $\Lambda(r, t) \in$ $\mathrm{SU}(2)$ with $\Lambda(r, 1)=-\Lambda(r, 0)$ and $\Lambda_{t}(r, 1)=-\Lambda_{t}(r, 0)$. This preserves the periodicity of $\left(\phi, a_{r}, a_{t}\right)$ as well as Eq. (1) and the boundary conditions. Clearly the fixed points of $\tau$ consist precisely of the fields which are onemonopole chains in disguise, i.e., periodic one-monopole solutions taken over two periods. There are exactly two such solutions, up to gauge equivalence: their Nahm-Hitchin data can be written as

$\phi=C\left(\mathrm{e}^{\pi s} \mp \mathrm{e}^{-\pi s}\right)\left[\cos (\pi t) \sigma_{2}+\sin (\pi t) \sigma_{3}\right]$,

$a_{r}=0, \quad a_{t}=(\mathrm{i} \pi / 2) \sigma_{1}$,

and they have $K= \pm 2$, respectively.

No other solutions of (1), with these boundary conditions, are known explicitly. One way of solving the equations numerically is by minimizing an "energy" functional, and we implemented this in order to get an idea of what the fields look like. Briefly, the details are as follows. Define

$$
\begin{aligned}
E_{L} & =\int_{-L}^{L} \int_{0}^{1} \mathcal{E} d t d r, \text { where } \\
\mathcal{E} & =\left|D_{1} \phi\right|^{2}+\left|D_{2} \phi\right|^{2}+|f|^{2}+\frac{1}{4}\left|\left[\phi, \phi^{*}\right]\right|^{2} .
\end{aligned}
$$

The $r$ cutoff $L$ has to be finite for the integral to converge, but in practice it does not have to be large since the solutions are well localized. Then there is a Bogomolny-type bound on $E_{L}$, and this bound is saturated if and only if the Hitchin equations (1) are satisfied. So, minimizing $E_{L}$ numerically gives a solution.

In the next section, we identify the four moduli in terms of the fields $\left(\phi, a_{\bar{s}}\right)$, at least in the asymptotic region of the moduli space. Then in Sec. III, we derive the asymptotic metric on $\mathcal{M}$ by direct calculation, and we see that it agrees with the metric previously derived by considering the forces between monopoles [4]. Section IV describes various geodesic surfaces in $\mathcal{M}$, and this is followed by a discussion of geodesics and the associated monopole dynamics in Sec. V.

\section{THE MODULI}

The moduli space $\mathcal{M}$ is four dimensional, and the aim here is to describe the four moduli in terms of the NahmHitchin data. Two of the moduli are the real and imaginary parts of the complex number $K$ appearing in the constraint (2). We shall describe the remaining two moduli in the asymptotic region of $\mathcal{M}$, which is where $|K| \gg 1$; this corresponds to the monopoles being widely separated. Numerical solutions, obtained as outlined above, indicate that the data $\left(\phi, a_{\bar{s}}\right)$ then resemble two well-separated lumps on the cylinder, located at the zeros $\pm s_{0}$ of det $\phi$. By this we mean that the gauge field $f$ is close to zero except at these two points, and the peaks at $s= \pm s_{0}$ become more concentrated as $|K|$ increases. In particular, since $f \approx 0$ in the central region $r \approx 0$, it makes sense to consider the $t$ holonomy there, and we define an angle $\theta$ by

$$
U_{0}=\mathcal{P} \exp \left(\int_{0}^{1} a_{t}(0, t) d t\right), \quad 2 \cos \theta=\operatorname{tr} U_{0} .
$$

The $x, y$ positions of the two monopoles are given by $x+\mathrm{i} y= \pm C \sqrt{-K},[3,5,6]$. As we shall see in the next section, their $z$ positions are $z= \pm \theta$. The sign ambiguity in the square root reflects the indistinguishability of the monopoles.

Equation (4) only determines $\theta$ up to a sign, but we may remove this ambiguity by using the Higgs field $\phi$, and regard $\theta \in(-\pi, \pi]$ as periodic with period $2 \pi$. In fact, $\theta$ is really a local coordinate on a $\mathbb{Z}_{2}$-twisted circle bundle over the asymptotic $K$ space, and the definition of its sign is a matter of choice. Here is one particular scheme, using the value $\phi_{0}$ of $\phi$ at $r=t=0$. The quantity $\xi=-\mathrm{i} \operatorname{tr}\left(U_{0} \phi_{0}\right)$ is a gauge-invariant complex number. If $\theta \neq 0, \pi$, then $\xi$ is nonzero, and it follows from $\operatorname{det} \phi_{0}=C^{2}(K-2) \approx C^{2} K$ that

(i) if $\operatorname{Re}(K) \geq 0$, then $\operatorname{Im}(\xi) \neq 0$, and we can define $\operatorname{sgn}(\theta)=\operatorname{sgn}(\operatorname{Im}(\xi))$;

(ii) if $\operatorname{Re}(K)<0$, then $\operatorname{Re}(\xi) \neq 0$, and we can define $\operatorname{sgn}(\theta)=\operatorname{sgn}(\operatorname{Re}(\xi))$.

If we write $K=|K| \mathrm{e}^{2 \pi \mathrm{i} \eta}$, then $\theta$ changes sign as $\eta$ goes from 0 to 1, i.e., as one goes around a loop in the asymptotic $K$ space. In fact, with this particular scheme, the jump occurs as $\eta$ crosses $1 / 4$. 
Finally, let us turn to the fourth modulus $\omega$, which corresponds to a relative phase between the two monopoles. It also corresponds to a relative phase between the two lumps on the cylinder. Define $f_{ \pm}=f\left( \pm s_{0}\right) \in \mathfrak{S H}(2)$ : in other words, the $f_{ \pm}$are the directions in the Lie algebra of the gauge field at the two peaks. Then we define $\omega$ to be the angle between $f_{+}$and $\hat{f}_{-}$, where $\hat{f}_{-}$is obtained by parallel propagating $f_{-}$from $-s_{0}$ to $s_{0}$ using $\partial_{\gamma} f=$ $-\left[a_{\gamma}, f\right]$, where $\gamma$ is a path between $-s_{0}$ and $s_{0}$. For large $|K|$ this formulation is path independent (up to winding around the cylinder) as the field strength vanishes between the peaks. This only defines $\omega$ up to a sign, but the ambiguity may be resolved as before, this time using the quantity $\xi=\mathrm{i} \operatorname{tr}\left(f_{+} f_{-} \phi_{0}\right)$. So $\omega \in(-\pi, \pi]$ has period $2 \pi$.

Since $f \rightarrow 0$ as $r \rightarrow \pm \infty$, it is also natural to consider the holonomies at infinity, namely,

$$
U_{ \pm}=\lim _{r \rightarrow \pm \infty} \mathcal{P} \exp \left(\int_{0}^{1} a_{t}(r, t) d t\right) .
$$

It follows from the equations and boundary conditions that $\operatorname{tr} U_{ \pm}=0$, so the $U_{ \pm}$individually contain no gaugeinvariant information. But the angle between them does: for example, let $\hat{U}_{-}$be the element of SU(2) obtained by parallel propagating $U_{-}$along $t=0$ from $r=-\infty$ to $r=\infty$, and define $\tilde{\omega}$ by $2 \cos \tilde{\omega}=\operatorname{tr}\left(U_{+} \hat{U}_{-}^{*}\right)$. This quantity $\tilde{\omega}$ is related to $\omega$ by $\tilde{\omega}-\omega=\pi$ (modulo integer multiples of $2 \pi$ ). By contrast with $\omega$, the definition of $\tilde{\omega}$ is valid throughout $\mathcal{M}$ and not just in the asymptotic region. In particular, for the special solutions (3), which are not in the asymptotic region, we can compute $U_{ \pm}$ exactly, and this shows that the fields (3) have $\tilde{\omega}=0$.

\section{THE ASYMPTOTIC METRIC}

The natural hyperkähler metric on $\mathcal{M}$ is believed to have no continuous symmetries, and it is not known explicitly. The asymptotic metric, however, has a fairly simple form. It was derived in [4] by studying the effective Lagrangian of the two-monopole system, in other words, the forces between well-separated monopoles. In this section, we see that this asymptotic metric can be calculated directly in terms of the Nahm-Hitchin data. In particular, this shows us how to identify the moduli of the previous section with the "monopole-based" moduli used in [4].

Let us think of a tangent vector in $\mathcal{M}$, at the point corresponding to the solution $\left(\phi, a_{\bar{s}}\right)$, as a perturbation $\left(\delta \phi, \delta a_{\bar{s}}\right)$ which preserves Eq. (1), and also satisfies the condition

$$
4\left\{D_{s}\left(\delta a_{\bar{s}}\right)+D_{\bar{s}}\left(\delta a_{s}\right)\right\}=\left[\phi, \delta \phi^{*}\right]+\left[\phi^{*}, \delta \phi\right]
$$

for the perturbation to be orthogonal to the gauge orbits at $\left(\phi, a_{\bar{s}}\right)$. Here $\delta a_{\bar{s}}=\frac{1}{2}\left(\delta a_{r}+\mathrm{i} \delta a_{t}\right)$ and $\delta a_{s}=\frac{1}{2}\left(\delta a_{r}-\right.$ $\left.\mathrm{i} \delta a_{t}\right)=-\left(\delta a_{\bar{s}}\right)^{*}$. The combined equations on $\left(\delta \phi, \delta a_{\bar{s}}\right)$ are equivalent to the pair

$$
D_{\bar{s}}(\delta \phi)=\left[\phi, \delta a_{\bar{s}}\right], \quad\left[\phi, \delta \phi^{*}\right]=4 D_{\bar{s}}\left(\delta a_{s}\right) .
$$

In addition to these differential equations (6), one also needs boundary conditions $\delta \phi \rightarrow 0, \delta a_{\bar{s}} \rightarrow 0$ as $r \rightarrow \infty$, and the constraint $\operatorname{tr}(\phi \delta \phi)=$ constant. The norm squared of the vector $V=\left(\delta \phi, \delta a_{\bar{s}}\right)$ is then defined to be

$\|V\|^{2}=\frac{1}{2} \operatorname{Re} \int \operatorname{tr}\left[(\delta \phi)(\delta \phi)^{*}+4\left(\delta a_{\bar{s}}\right)\left(\delta a_{\bar{s}}\right)^{*}\right] d r d t$,

and this gives the metric on $\mathcal{M}$.

Note that if $V_{1}=\left(\delta_{1} \phi, \delta_{1} a_{\bar{s}}\right)$ is one solution of (6), then so are each of

$$
\begin{aligned}
& V_{2}=\left(\delta_{2} \phi, \delta_{2} a_{\bar{s}}\right)=\left(\mathrm{i} \delta_{1} \phi, \mathrm{i} \delta_{1} a_{\bar{s}}\right), \\
& V_{3}=\left(\delta_{3} \phi, \delta_{3} a_{\bar{s}}\right)=\left(2 \delta_{1} a_{s}, \frac{1}{2} \delta_{1} \phi^{*}\right), \\
& V_{4}=\left(\delta_{4} \phi, \delta_{4} a_{\bar{s}}\right)=\left(2 \mathrm{i} \delta_{1} a_{s}, \frac{1}{2} \mathrm{i} \delta_{1} \phi^{*}\right) .
\end{aligned}
$$

Furthermore, these four vectors are orthogonal with the same norm; in other words, $\left\langle V_{a}, V_{b}\right\rangle=p^{2} \delta_{a b}$ for some real constant $p$. Suppose that $V_{1}$ corresponds to the increments $\left(\delta_{1} K_{r}, \delta_{1} K_{i}, \delta_{1} \theta, \delta_{1} \omega\right)$ in the moduli, where $K=K_{r}+\mathrm{i} K_{i}$ are the real and imaginary parts of $K$, and similarly for the other $V_{a}$. Define the $4 \times 4$ matrix $Q$ by

$$
Q=p^{-1}\left[\begin{array}{cccc}
\delta_{1} K_{r} & \delta_{2} K_{r} & \delta_{3} K_{r} & \delta_{4} K_{r} \\
\delta_{1} K_{i} & \delta_{2} K_{i} & \delta_{3} K_{i} & \delta_{4} K_{i} \\
\delta_{1} \theta & \delta_{2} \theta & \delta_{3} \theta & \delta_{4} \theta \\
\delta_{1} \omega & \delta_{2} \omega & \delta_{3} \omega & \delta_{4} \omega
\end{array}\right] .
$$

Then the coefficients of the metric on $\mathcal{M}$, with respect to the local coordinates $\left(K_{r}, K_{i}, \theta, \omega\right)$, are the entries in the matrix $g=\left(Q Q^{t}\right)^{-1}$.

In the asymptotic region $|K| \gg 1$, there is a crude but effective approximate solution $\left(\phi, a_{\bar{s}}\right)$, namely,

$$
\phi=C \sqrt{H} \sigma_{3}, \quad a_{t}=\mathrm{i} \theta \sigma_{3}, \quad a_{r}=0,
$$

where $H=2 \cosh (2 \pi s)-K$. The branch cuts in (8) are gauge removable, and these fields provide a solution everywhere except at the two singular points $s= \pm s_{0}$. Numerics indicates that it is a good approximation, for large $|K|$, to the actual smooth solutions, except very close to the singular points. The gauge field consists, in effect, of delta functions at the singular points, and we complete the approximate description of the field by simply assigning elements $f_{ \pm}$of $\mathfrak{S H}(2)$, orthogonal to $\sigma_{3}$, to these two points. The moduli $K_{r}, K_{i}$ and $\theta$ appear explicitly in (8), and $\omega$ is the angle between $f_{+}$and $\hat{f}_{-}$.

To keep things simple in what follows, let us restrict to the case $\operatorname{Re}(K)>0$. Since $\theta$ and $\omega$ are twisted rather than global coordinates, one obtains the complete picture by using the case $\operatorname{Re}(K)<0$ as well, and then patching things together in an appropriate way.

Let $V_{1}$ be the perturbation $\delta_{1} \phi=\frac{1}{2} \varepsilon h \sigma_{3}, \delta_{1} a_{\bar{s}}=0$, where $h=h(s)=H^{-1 / 2}$, and $\varepsilon$ is a small parameter. This satisfies (6) and the associated boundary conditions, 
except at the singularities. Note that $\left\|V_{1}\right\|^{2}=p^{2}=\varepsilon^{2} I$, where

$$
I=\frac{1}{4} \int \frac{d r d t}{|H|}=\frac{1}{4} \int \frac{d r d t}{|2 \cosh (2 \pi s)-K|} .
$$

The corresponding variations in the moduli are $\delta_{1} K_{r}=$ $\varepsilon C^{-1}$ and $\delta_{1} K_{i}=\delta_{1} \theta=\delta_{1} \omega=0$. Following the pattern described above, the next perturbation $V_{2}$ is $\delta_{2} \phi=\frac{1}{2} \mathrm{i} \varepsilon h \sigma_{3}$, $\delta_{2} a_{\bar{s}}=0$, and this has $\delta_{2} K_{i}=\varepsilon C^{-1}, \delta_{2} K_{r}=\delta_{2} \theta=$ $\delta_{2} \omega=0$.

For $V_{3}$ we get $\delta_{3} \phi=0$, whence $\delta_{3} K_{r}=\delta_{3} K_{i}=0$; and $\delta_{3} a_{\bar{s}}=\frac{1}{4} \varepsilon \bar{h} \sigma_{3}$. Thus $\delta_{3} a_{r}=-\frac{1}{2} \mathrm{i} \varepsilon \operatorname{Im}(h) \sigma_{3}$ and $\delta_{3} a_{t}=-\frac{1}{2}$ i $\varepsilon \operatorname{Re}(h) \sigma_{3}$, from which one directly computes $\delta_{3} \theta=-\frac{1}{2} \varepsilon \operatorname{Re}\left(h_{0}\right)$, where

$$
h_{0}=\int_{0}^{1} h(0, t) d t \approx 1 / \sqrt{-K} .
$$

The perturbation $V_{3}$ does not affect $f_{ \pm}$(this has been checked with numerical examples), so a variation of $\omega$ arises only from the change of $a$ along the path between $-s_{0}$ and $s_{0}$. We thus obtain $\delta_{3} \omega=-2 \varepsilon[\operatorname{Im}(J)+\operatorname{Re}(L)]$, where

$$
J=\int_{0}^{r_{0}} h\left(r, t_{0}\right) d r, \quad L=\int_{0}^{\eta} h(0, t) d t \approx h_{0} \eta
$$

[recall that $\eta \in[0,1)$ is defined by $\left.K=|K| \mathrm{e}^{2 \pi \mathrm{i} \eta}\right]$. Similarly, for $V_{4}$ we have $\delta_{4} \phi=0$ and $\delta_{4} a_{\bar{s}}=\frac{1}{4}$ i $\varepsilon \bar{h} \sigma_{3}$; and the corresponding variations in the moduli are $\delta_{4} K_{r}=$ $\delta_{4} K_{i}=0, \quad \delta_{4} \theta=-\frac{1}{2} \varepsilon \operatorname{Im}\left(h_{0}\right)$ and $\delta_{4} \omega=2 \varepsilon[\operatorname{Re}(J)-$ $\operatorname{Im}(L)]$. Note that the "knock-on" effect of one perturbation on another has been ignored, and indeed for large $|K|$ such terms only make relatively small contributions to the metric.

The next step is to compute the leading terms in the integrals $I$ and $J$ for $|K| \gg 1$. These are obtained from the approximation $1 / H(r, t) \approx-1 / K$ for $0 \leq r<r_{0}$, $1 / H(r, t) \approx 0$ for $r>r_{0}$, which gives

$$
I \sim \frac{\log |K|}{4 \pi|K|}, \quad J \sim \frac{\log |K|}{2 \pi \sqrt{-K}}
$$

as $|K| \rightarrow \infty$. Then it is straightforward to calculate the asymptotic metric as described above, via the matrix $Q$, and we get

$d s^{2}=\frac{\log |K|}{4 \pi|K|}\left(C^{2}|d K|^{2}+4|K| d \theta^{2}\right)+\frac{\pi}{\log |K|}(d \omega-4 \eta d \theta)^{2}$.

Now the asymptotic metric $d s_{C K}^{2}$ of [4], which was computed by considering the forces between monopoles, is given by

$$
\frac{1}{4 \pi} d s_{C K}^{2}=4 U\left(d x^{2}+d y^{2}+d z^{2}\right)+U^{-1}(d \nu+g d z)^{2},
$$

where $(x, y, z)$ is the location of one of the monopoles relative to the center of mass, and $\nu$ is a relative phase with period $\pi$. The functions $U$ and $g$ are defined by

$$
U=\frac{1}{\pi} \log \rho, \quad g=2\left(\chi-\chi_{0}\right),
$$

where $x+\mathrm{i} y=\rho \mathrm{e}^{\mathrm{i} \pi\left(\chi-\chi_{0}\right)}$, with $\chi_{0}$ being some constant. We already know [3,5] that $x+\mathrm{i} y=C \sqrt{-K}$, and it is straightforward to check that the metrics (10) and (11) agree, up to an overall factor of $8 \pi$, if we make the identification $\theta=z$ and $\omega=-2 \nu$. In particular, therefore, $2 \theta$ is the $z$ offset of the monopoles.

\section{GEODESIC SURFACES}

The geometry of the moduli space $\mathcal{M}$, and, in particular, its geodesics, corresponds to the dynamics of monopole systems in situations where radiative losses are small and, in particular, at small speeds [7,8]. Not knowing the metric explicitly means that we cannot find many geodesics exactly, but some can be obtained as fixed-point sets of discrete symmetries of $\mathcal{M}$. The first step, as in the familiar $\mathbb{R}^{3}$ case [9], is to identify geodesic surfaces in $\mathcal{M}$; we do this by looking for discrete symmetries of the system (1) and (2). The most obvious symmetry is

$$
\phi \mapsto-\phi, \quad a_{\bar{s}} \mapsto a_{\bar{s}} .
$$

In the monopole picture, Eq. (12) corresponds to rotation by $\pi$ about the $z$ axis. Let $\mathcal{S}$ denote the fixed-point set of (12). Since $K$ is preserved, Eq. (12) acts only on the other two moduli $\theta$ and $\tilde{\omega}$ (for this section, it is more convenient to use $\tilde{\omega}$ than $\omega$ ). From the discussion of signs in Sec. II, it is clear that the effect of (12) is $\theta \mapsto-\theta$ and $\tilde{\omega} \mapsto-\tilde{\omega}$. Thus, asymptotically, $\mathcal{S}$ has four disconnected components, corresponding to $\theta, \tilde{\omega} \in\{0, \pi\}$. In this asymptotic regime, we see a pair of monopoles, located at the points $x+\mathrm{i} y= \pm C \sqrt{-K}, z= \pm \theta$, and with their phases either aligned or anti-aligned depending on $\tilde{\omega}$.

The question now is what $\mathcal{S}$ looks like globally, not just asymptotically. As described in [6], the solutions belonging to $\mathcal{S}$ take a simplified form: there exists a gauge in which

$$
a_{\bar{s}}=h \sigma_{1}, \quad \phi=\frac{1}{2}(f+g) \sigma_{2}+\frac{1}{2} \mathrm{i}(f-g) \sigma_{3},
$$

where $f, g$ and $h$ are complex-valued functions. The constraint on $\operatorname{det} \phi$ is $f g=C^{2} H=C^{2}[2 \cosh (2 \pi s)-K]$, and the Hitchin equations become

$$
\Delta \log |f|=|f|^{2}-C^{4}|H / f|^{2},
$$

together with $2 h=-\partial_{\bar{s}} \log f$, where $\Delta=4 \partial_{s} \partial_{\bar{s}}$ is the Laplacian. The boundary condition is $\left|f^{2} / H\right| \rightarrow C^{2}$ as $r \rightarrow \pm \infty$, and the solutions have the symmetry

$$
|f(-r, 1-t)|=|f(r, t)| \text { for all } r, t .
$$


The remaining gauge freedom consists of

$$
\begin{aligned}
& \{f \mapsto g, g \mapsto f, h \mapsto-h\} \quad \text { and } \\
& \quad\left\{f \mapsto \lambda^{-2} f, g \mapsto \lambda^{2} g, h \mapsto h+\partial_{\bar{s}} \lambda\right\},
\end{aligned}
$$

where $\lambda(r, t)$ is periodic and of unit modulus.

Taking account of this gauge freedom, it is easy to see that there are four classes of solutions of (13): $f$ could have one zero or none; and $\lim _{r \rightarrow \infty} \operatorname{Im} \log f(r, t)=2 \pi n t$, where $n$ is either 0 or 1 . As we shall see below, these four possibilities correspond to the four asymptotic components of $\mathcal{S}$. Let us begin by studying one of these cases in detail, namely, where $f$ has no zeros and $n=0$. In particular, this means that $f$ has the form

$$
f=\mathrm{e}^{\psi / 2},
$$

where $\psi$ is a complex-valued periodic function. If we write $\psi=\alpha+\mathrm{i} \beta$ for the real and imaginary parts of $\psi$, then $a_{t}=\frac{1}{4} \mathrm{i}\left(\alpha_{r}-\beta_{t}\right) \sigma_{1}$, and therefore the holonomy at $r$ is

$$
U_{r}=\exp \left[\frac{\mathrm{i}}{4} \int_{0}^{1} \alpha_{r}(r, t) d t \sigma_{1}\right]
$$

The boundary condition says that $\alpha(r, t) \sim \pm 2 \pi r$ as $r \rightarrow \pm \infty$, so the holonomies at the two ends are $U_{ \pm}=$ $\pm \mathrm{i} \sigma_{1}$, and hence $\tilde{\omega}=\pi$. To compute $\theta$, note that the symmetry (14) implies $\alpha_{r}(0,1-t)=-\alpha_{r}(0, t)$; it follows that $U_{0}=1$ and so $\theta=0$.

For $f$ of the form (15), Eq. (13) becomes

$$
\Delta \alpha=2\left(\mathrm{e}^{\alpha}-C^{4}|H|^{2} \mathrm{e}^{-\alpha}\right),
$$

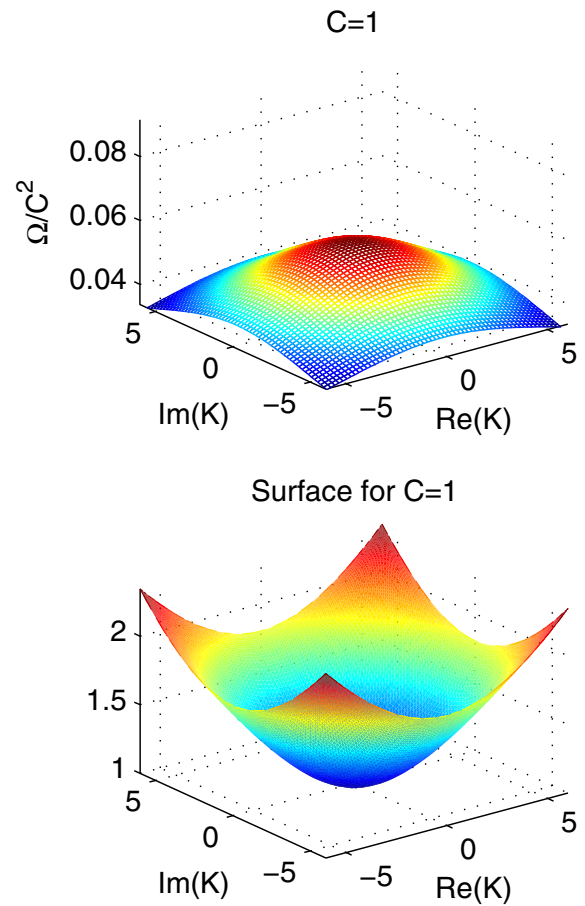

which has a unique solution for each value of $K \in \mathbb{C}$. So this case gives one of the components $\mathcal{S}_{-}$of $\mathcal{S}$, namely, the one corresponding to $\theta=0, \tilde{\omega}=\pi$; and $\mathcal{S}_{-}$is diffeomorphic to $\mathbb{C}$. With its natural metric (the restriction of the metric on $\mathcal{M}$ ), the surface $\mathcal{S}_{-}$is a deformed version of the Atiyah-Hitchin cone [8,9], having no continuous symmetries (unlike the Atiyah-Hitchin cone itself, which is rotationally symmetric). The metric on $\mathcal{S}_{-}$may be calculated numerically, and this is instructive in that it shows the effect of varying the parameter $C$. The procedure is as follows.

Given complex numbers $K$ and $\delta K$, with $\delta K$ small, we solve (16) numerically for $K$ and for $K^{\prime}=K+\delta K$, giving real functions $\alpha$ and $\alpha^{\prime}$, respectively. This may be done by minimizing an appropriate functional of $\alpha$, as described in [6]. We take $\beta=0$, so $\psi=\alpha$ is real valued: this is just a gauge choice. However, setting $\beta^{\prime}=0$ as well leads to a perturbation $\left(\delta \phi, \delta a_{\bar{s}}\right)$ which does not satisfy the gaugeorthogonality condition (5). So we need to use $\delta \psi=\alpha^{\prime}-$ $\alpha+\mathrm{i} \delta \beta$, where $\delta \beta$ is determined by the requirement that $\delta \psi$ is orthogonal to the gauge orbits at $\psi=\alpha$. This is just a linear equation for $\delta \beta$, having a unique solution, and it is straightforward to solve numerically. The final step then uses (7) to evaluate $\|\delta K\|^{2}$, and hence gives the metric on $\mathcal{S}_{-}$. This metric has the form $d s^{2}=\Omega(K)|d K|^{2}$, and we know from (10) that

$$
\Omega(K) \sim \frac{C^{2}}{4 \pi|K|} \log |K| \quad \text { as }|K| \rightarrow \infty .
$$

The upper plots in Fig. 1 show $\Omega(K) / C^{2}$ on $|K|<6$, for $C=1$ and $C=5$, respectively. The corresponding lower plots are rough sketches of the surface, obtained by

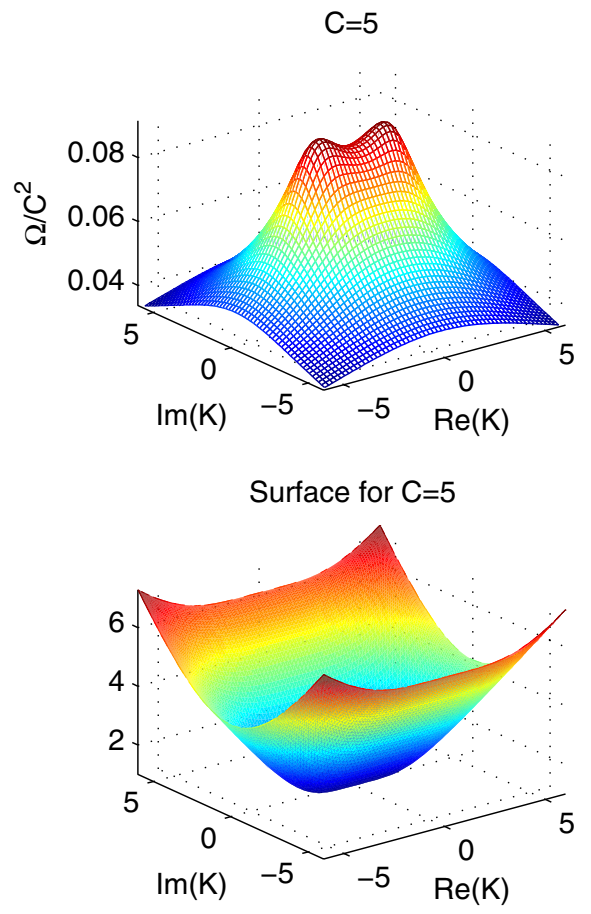

FIG. 1 (color online). The metric factor $\Omega / C^{2}$ on the geodesic surface $\mathcal{S}_{-}$, and sketches of the surface, for $C=1$ and $C=5$. 
computing the Gaussian curvature numerically from $\Omega$ and then finding an embedded surface in $\mathbb{R}^{3}$ with that curvature. We see that for $C=1$, the surface has approximate rotational symmetry, as one would expect since it should approach the Atiyah-Hitchin cone as $C \rightarrow 0$. For larger $C$, however, the lack of symmetry becomes apparent, with the cone becoming stretched in the $K_{r}$ direction.

We next consider the component of $\mathcal{S}$ corresponding to the case where $f$ has no zeros and $n=1$. The corresponding geodesic surface $\mathcal{S}_{+}$is isometric to $\mathcal{S}_{-}$: in fact, the isometry is the map $\tau$. The action of $\tau$ amounts to gauging by the antiperiodic transformation $\Lambda=\exp \left(\mathrm{i} \pi t \sigma_{3}\right)$, and the effect of this on the moduli is $K \mapsto K, \theta \mapsto \theta+\pi$, $\tilde{\omega} \mapsto \tilde{\omega}$. So, in particular, $\mathcal{S}_{+}$has $\theta=\tilde{\omega}=\pi$.

For the two remaining cases, $f$ has a zero, which has to be one of the zeros of $H$. Then $f$ has either of the two forms

$$
\begin{gathered}
f=C \mathrm{e}^{\psi / 2} \mu \mathrm{e}^{\mathrm{i} \pi t}, \\
f=C \mathrm{e}^{\psi / 2} \mu \mathrm{e}^{-\mathrm{i} \pi t},
\end{gathered}
$$

where

$$
\mu=\mathrm{e}^{\pi s}-W \mathrm{e}^{-\pi s}, \quad W=\left(K+\sqrt{K^{2}-4}\right) / 2,
$$

and we take the branch of the square root such that $|W|>1$. The boundary condition is $\operatorname{Re}(\psi) \rightarrow 0$ as $r \rightarrow \infty$, $\operatorname{Re}(\psi) \rightarrow-2 \log |W|$ as $r \rightarrow-\infty$. These classes (17) and (18) both have $\tilde{\omega}=0$, and they have $\theta=0, \pi$, respectively. They are interchanged by the map $\tau$. However, each class contains the two special solutions (3), which are the fixed points of $\tau$. So, in fact, we get a single component $\mathcal{S}_{0}$ of $\mathcal{S}$, consisting of two copies of the $K$ plane branched over the points $K= \pm 2$. In effect, interchanging the two branches of the square root in (19) interchanges the forms (17) and (18). The single surface $\mathcal{S}_{0}$ has two asymptotic regions, each of which is conelike: so the picture may be described as a double trumpet, in contrast to the Atiyah-Hitchin trumpet of the $\mathbb{R}^{3}$ case [9]. The metric on $\mathcal{S}_{0}$ has no continuous symmetries, but it has an approximate rotational symmetry (about the axis of the trumpet) for small $C$.

The second expression in (19) is just the usual conformal mapping $K=W+W^{-1}$, and this gives us a global coordinate $W \in \mathbb{C}^{*}$ on $\mathcal{S}_{0}$. Given $W$, we take the field to be determined by (18). If $|W| \gg 1$ it lies on the sheet $\theta=\pi$, while if $|W| \ll 1$ it lies on the sheet $\theta=0$. The $K$ plane is cut on the line segment $-2 \leq K \leq 2$, which corresponds to $|W|=1$, and crossing this line takes us from one sheet to the other. In the next section, we shall describe geodesics which cross sheets in this way.

Finally, we remark on the large- $C$ behavior of the metric. In [5] it was suggested that when $C \gg 1$, i.e., when the monopoles are large compared to the $z$ period, the only relevant modulus is $K$; and the metric on the $K$ plane was computed by using an approximation to the monopole fields which is valid in this limit. In fact, we can also compute this limiting metric in the Nahm-transformed picture. The crucial observation is that in the large- $C$ regime, the fields are

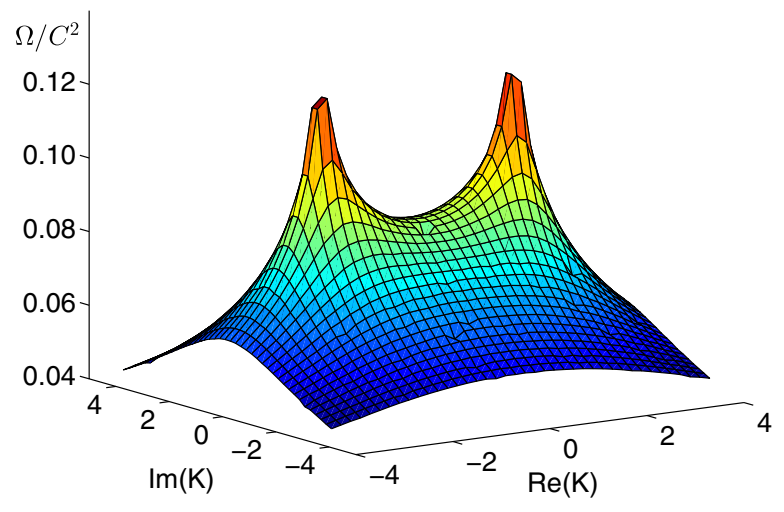

FIG. 2 (color online). Plot of the large- $C$ conformal factor $I(K)$. The peaks are at $K= \pm 2$.

well approximated by the singular solution (8), except at its singularities. In other words, the large- $|K|$ and large- $C$ approximations are the same. It follows that the large- $C$ metric on each component of $\mathcal{S}$ is $d s^{2}=C^{2} I|d K|^{2}$, where $I(K)$ is defined in (9). In Sec. III we only used $I(K)$ for $|K|$ large, but in this context we need it for all $K \in \mathbb{C}$. The integral (9) converges for all $K$ except the two special values $K= \pm 2$, and its value is plotted in Fig. 2, as a function of $K \in \mathbb{C}$. This picture should be compared with the plots of $\Omega / C^{2}$ in Fig. 1 for $C=1$ and $C=5$ : the function $I(K)$ appears to be the $C \rightarrow \infty$ limit of $\Omega / C^{2}$. A more extensive numerical investigation of $\Omega$, for a wider range of $C$, bears this out. Note also that in this limit, the surface $\mathcal{S}_{0}$ resembles two copies of Fig. 2, branched between the singularities.

\section{GEODESICS AND MONOPOLE SCATTERING}

Our aim in this section is to identify geodesics on $\mathcal{S}_{ \pm}$ and $\mathcal{S}_{0}$, and to interpret these in terms of two-monopole trajectories. One could construct such geodesics numerically, for example, by using the numerically derived metrics on these surfaces; such a construction was implemented in [5] in the large- $C$ limit. But here we will do something more analytic, namely, identify geodesics on $\mathcal{S}_{ \pm}$and $\mathcal{S}_{0}$ as fixed-point sets of additional symmetries of the system. An example of this type was presented in [6]; here we will give a fuller discussion, revealing rather more interesting behavior than was seen before.

The first step is to describe the relevant symmetries of the Hitchin equations (1). There are two of them, namely,

$$
\begin{aligned}
K & \mapsto \bar{K}, \quad \phi(r, t) \mapsto \phi(r, 1-t)^{*}, \\
a_{r}(r, t) & \mapsto a_{r}(r, 1-t), \quad a_{t}(r, t) \mapsto-a_{t}(r, 1-t), \\
K & \mapsto-\bar{K}, \quad \phi(r, t) \mapsto \mathrm{i} \phi\left(r, \frac{1}{2}-t\right)^{*}, \\
a_{r}(r, t) & \mapsto a_{r}\left(r, \frac{1}{2}-t\right), \quad a_{t}(r, t) \mapsto-a_{t}\left(r, \frac{1}{2}-t\right) .
\end{aligned}
$$

The fixed-point sets of (20) and (21) are geodesic hypersurfaces in $\mathcal{M}$ given by $K \in \mathbb{R}$ and $K \in \mathrm{i} \mathbb{R}$, respectively, 

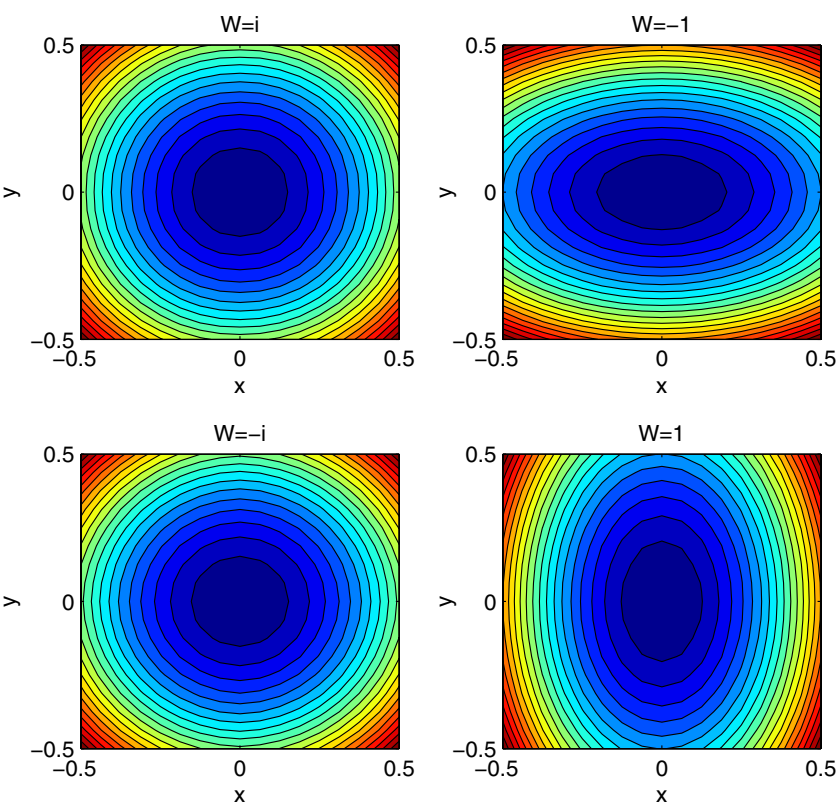

FIG. 3 (color online). Contour plots of $|\Phi|^{2}$ on $z=\pi / 2$, for four points on the geodesic $|W|=1$.

and the intersections of these with the surfaces $\mathcal{S}_{ \pm}$and $\mathcal{S}_{0}$ are geodesics. For $\mathcal{S}_{ \pm}$, this leads to a picture which is essentially the same as in the $\mathbb{R}^{3}$ case: the geodesics pass over the "center" of the deformed cone, and this corresponds to $90^{\circ}$ planar scattering of two monopoles, via a toroidal twomonopole solution. The geodesics on the double trumpet $\mathcal{S}_{0}$ are more interesting, however, and we shall focus on them in what follows.

In terms of the coordinate $W \in \mathbb{C}^{*}$ on $\mathcal{S}_{0}$, the symmetries (20) and (21) lead to five complete geodesics, namely, the four half-axes in the $W$ plane and the unit circle $|W|=1$. The last of these is a closed geodesic which winds around the waist of the double trumpet. The two points $W= \pm 1$ on it are the two special solutions (3) representing onemonopole fields taken over two periods, the monopoles being located on the $z$ axis at $z= \pm \pi / 2$. In fact, the geodesic consists entirely of monopole pairs located at these two points on the $z$ axis: the monopoles stay in the same position and simply oscillate in shape. This is illustrated in Fig. 3, which was generated by the numerical solution of the Hitchin equations (13) for a range of $W$ values, followed by numerical implementation of the inverse Nahm transform. The parameter $C$ was taken to have the value $C=1$. Each of the plots in Fig. 3 is a contour plot of $|\Phi|^{2}$ on the plane $z=\pi / 2$, with the Higgs field $\Phi$ having a zero at the center, $x=y=0$. The cases $W= \pm \mathrm{i}$ correspond to $K=0$ on each of the two $K$ sheets, and one then has an additional $x \leftrightarrow y$ symmetry which is absent for the other points on this bounded trajectory. The pictures on the plane $z=-\pi / 2$ are the same. So we have a periodic trajectory representing a string of equally spaced monopoles, with all their kinetic energy coming from their in-phase shape oscillation.

The other four geodesics mentioned above are of scattering type, where two widely separated monopoles undergo a head-on collision and then separate again. Let us first describe the $W>0$ case, i.e., $W$ on the positive real axis. A point with $0<W \ll 1$ corresponds to a pair of monopoles widely separated on the $y$ axis, in fact, at $x=0=z, y=$ $\pm C / \sqrt{W}$. At the other end of the geodesic, where $W \gg 1$, we have monopoles at $x=0, y= \pm C \sqrt{W}, z=\pi$. In other words, the monopoles approach each other along the $y$ axis, collide, and emerge along the $\pm y$ directions but shifted by half a period in $z$. Using a numerical Nahm transform for a sequence of real $W$ values near $W=1$ reveals what happens to the monopoles as they collide: the results of this are shown
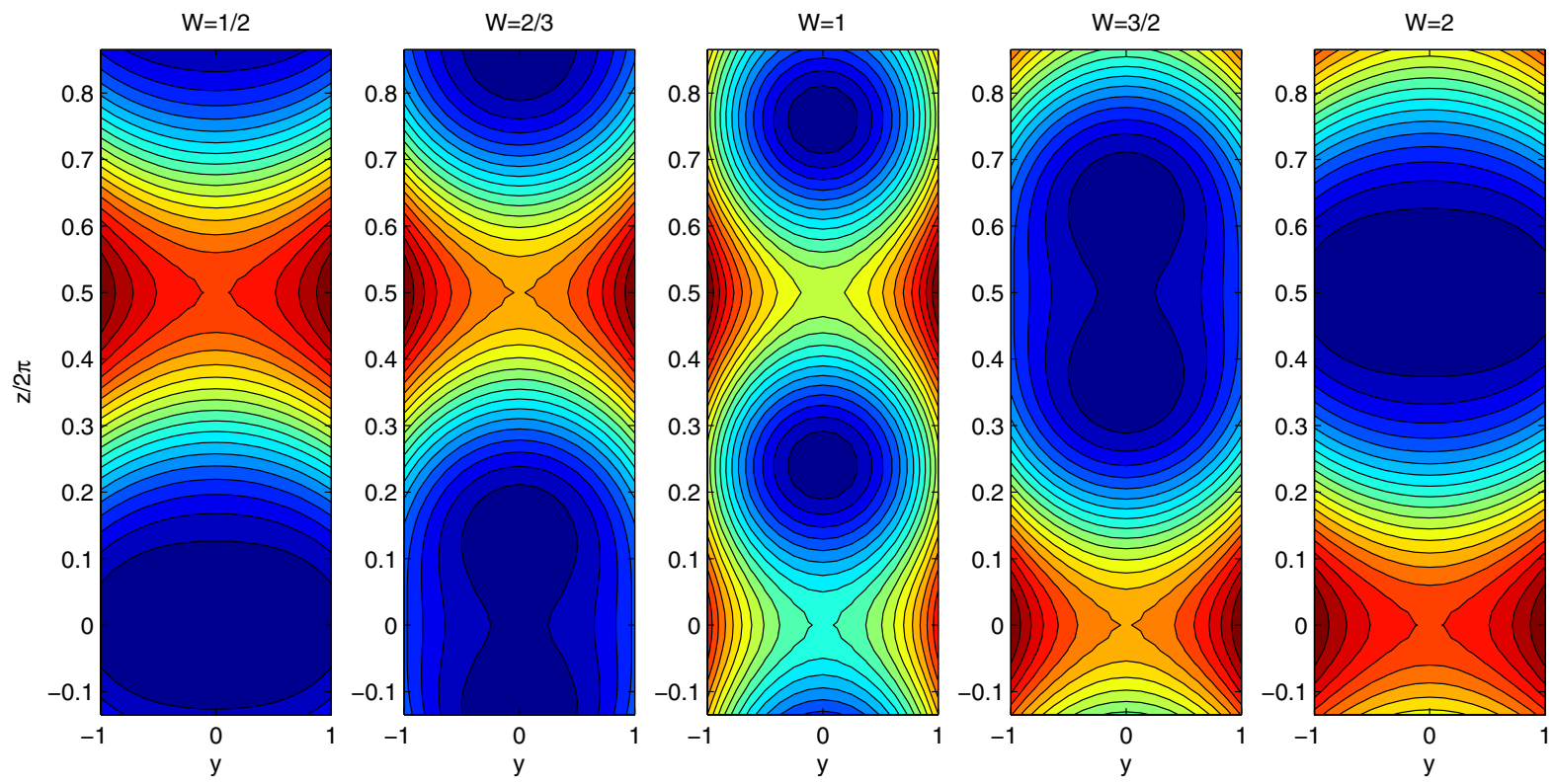

FIG. 4 (color online). Contour plots of $|\Phi|^{2}$ on $x=0$, for five points on the geodesic $W>0$. 
in Fig. 4. In effect, the scattering takes place in the plane $x=0$, and so we use contour plots in this plane. Note that the range of $z$ in the plots has been shifted, using the periodicity, in order to give a clearer representation of the scattering. As $W$ increases from a small positive value, the monopoles come in along the $y$ axis, with $z=0$. By $W=1 / 2$ (the first plot in Fig. 4) we see that they have merged at the origin. Then (second plot, with $W=2 / 3$ ) they begin to separate along the $z$ axis. At $W=1$, they are equidistant: this is a special solution (3). Then they re-merge at $z=\pi$ on the $z$ axis, and separate in the $\pm y$ directions. The combination of $90^{\circ}$ scattering in the $y z$ plane and periodicity in $z$ leads, in this example, to a picture in which monopoles emerge in the same directions as they entered, but shifted by half a period. The plots in Fig. 4 are for $C=1$; for other values of $C$, the picture is qualitatively the same, although the details differ-for example, the value of $W$ at which the monopoles merge.

Let us consider, next, the geodesic $W=\mathrm{i} p^{2}$ with $p>0$, i.e., $W$ on the positive imaginary axis. If $p \gg 1$, then $K \approx$ $\mathrm{i} p^{2}$ and the monopoles are located at $x+\mathrm{i} y= \pm C p \mathrm{e}^{-\mathrm{i} \pi / 4}$, $z=\pi$; while if $0<p \ll 1$, then $K \approx-\mathrm{i} p^{2}$ and the monopoles are located at $x+\mathrm{i} y= \pm C p^{-1} \mathrm{e}^{\mathrm{i} \pi / 4}, z=0$. So here the trajectory is fully three dimensional: the monopoles undergo right-angle scattering in the $x y$ direction, as well as being shifted by half a period in $z$.

As long as $C$ is not too large, any radial line in the $W$ plane is an approximate geodesic representing head-on scattering of two monopoles, and it is easy to see that we get a picture which interpolates between the two examples above. In fact, the line $W=p^{2} \mathrm{e}^{\mathrm{i} \nu}$, with $\nu$ fixed, gives a scattering angle of $\nu$, in addition to the $z$ shift. For large $C$, however, one gets rather different trajectories: see, for example, Fig. 6 of [5].

\section{CONCLUDING REMARKS}

We have seen that the periodic monopole system admits dynamical behavior not seen in the nonperiodic $\mathbb{R}^{3}$ case, in particular, head-on collision of two monopoles resulting in scattering through any angle, accompanied by a halfperiod shift. This is a consequence both of the periodicity and of the absence of rotational symmetry about the periodic axis. It would be worth studying other geodesics on the double-trumpet geodesic surface, not just those representing head-on collisions, as this might reveal further novel behavior.

We have shown that the asymptotic metric on the twomonopole moduli space may be computed directly via a simple approximation of the relevant $\mathrm{Nahm}$-Hitchin data, and this metric agrees, as expected, with that derived by considering the effective two-monopole Lagrangian. This asymptotic metric is relatively simple, having continuous symmetries; in particular, one can identify geodesic surfaces which are different from those described in this paper, and which do not involve $\theta$ and $\omega$ remaining constant. It would be interesting to investigate the global structure of these and to look for geodesics (trajectories) where $\theta$ and/or $\omega$ change.

The methods used here should extend to the case of higher charge monopoles. In the $\mathbb{R}^{3}$ case, it is particularly useful to consider multimonopoles invariant under discrete subgroups of the rotation group $[8,10]$. Because of the lack of rotational symmetry in the periodic case, it seems unlikely that the full scope of this technique could be applied. But certain discrete symmetries such as cyclic symmetry should remain relevant, and it would be worth making a systematic study of the symmetries of the $\mathrm{SU}(n)$ Hitchin system, corresponding to centered periodic $n$-monopole solutions, for higher values of $n$. Some preliminary results along these lines have been obtained, and further work is in progress.

\section{ACKNOWLEDGMENTS}

Both authors were supported by the UK Science and Technology Facilities Council. For R. S. W. this was by means of the Consolidated Grant No. ST/J000426/1.
[1] P. J. Braam and P van Baal, Commun. Math. Phys. 122, 267 (1989).

[2] H. Schenk, Commun. Math. Phys. 116, 177 (1988).

[3] S. Cherkis and A. Kapustin, Commun. Math. Phys. 218, 333 (2001).

[4] S. A. Cherkis and A. Kapustin, Phys. Rev. D 65, 084015 (2002).

[5] R. Maldonado, J. High Energy Phys. 02 (2013) 099.

[6] D. Harland and R. S. Ward, Phys. Lett. B 675, 262 (2009).
[7] N. S. Manton, Phys. Lett. 110B, 54 (1982).

[8] N.S. Manton and P.M. Sutcliffe, Topological Solitons (Cambridge University Press, Cambridge, England, 2004).

[9] M.F. Atiyah and N.J. Hitchin, The Geometry and Dynamics of Magnetic Monopoles (Princeton University Press, Princeton, 1988).

[10] N. J. Hitchin, N.S. Manton, and M. K. Murray, Nonlinearity 8, 661 (1995). 